\title{
Association between Serum $\gamma$-Glutamyltransferase and Chronic Kidney Disease among US Adults
}

\author{
Srinivas Teppala ${ }^{a}$ Anoop Shankar ${ }^{a}$ Jialiang Li ${ }^{b}$ Tien Yin Wong ${ }^{b, c}$ \\ Alan Ducatman ${ }^{a}$ \\ ${ }^{a}$ Department of Community Medicine, West Virginia University School of Medicine, Morgantown, W. Va., USA; \\ ${ }^{b}$ Singapore Eye Research Institute, National University of Singapore, Singapore; ${ }^{C}$ Centre for Eye Research Australia, \\ University of Melbourne, Melbourne, Vic., Australia
}

\section{Key Words}

$\gamma$-Glutamyltransferase $\cdot$ Chronic kidney disease $\cdot$ NHANES

\begin{abstract}
Background/Aim: Serum $\gamma$-glutamyltransferase (GGT), a biomarker of oxidative stress, is associated with an increased risk of diabetes and hypertension. However, it is not known whether higher serum GGT is independently associated with chronic kidney disease (CKD). Therefore, we examined the association between serum GGT levels and CKD in a representative sample of US adults. Methods: We conducted a cross-sectional study of 9,516 National Health and Nutrition Examination Survey 1999-2002 participants $\geq 18$ years of age (52.40\% women). Serum GGT was categorized into quartiles for the analysis. CKD $(n=622)$ was defined as an estimated glomerular filtration rate of $<60 \mathrm{ml} / \mathrm{min} / 1.73 \mathrm{~m}^{2}$. $\boldsymbol{R e}$ sults and Conclusion: Higher serum GGT levels were not associated with CKD after adjusting for age, sex, race/ethnicity, education levels, smoking, alcohol intake, body mass index (BMI), diabetes, hypertension and serum cholesterol. Compared to quartile 1 of GGT, the odds ratio ( $95 \%$ confidence interval) of CKD associated with quartile 4 was 1.02 (0.811.28); $p$ trend $=0.377$. Subgroup analyses that examined the
\end{abstract}

relation between GGT and CKD by gender, alcohol intake, and $\mathrm{BMI}$ categories also showed a consistent null association. In summary, there was no association between increasing levels of serum GGT and CKD in a sample of US adults.

Copyright $\odot 2010$ S. Karger AG, Basel

\section{Introduction}

$\gamma$-Glutamyltransferase (GGT) is a cell-surface enzyme that plays a major role in the extracellular catabolism of glutathione (GSH), the main antioxidant in mammalian cells $[1,2]$. Serum levels of GGT may therefore be considered a marker of oxidative stress [3]. Previous epidemiological studies have reported associations between elevated GGT levels and peripheral arterial disease $[4,5]$ and cardiovascular disease [6]. Studies have also shown that higher GGT levels are related to diabetes mellitus $[7,8]$ and hypertension $[8,9]$, which are risk factors for chronic kidney disease (CKD). However, few studies have examined the association between GGT levels and CKD. We examined the association between serum GGT levels and CKD in participants of the National Health and $\mathrm{Nu}$ trition Examination Survey (NHANES) 1999-2002, a

\section{KARGER}

Fax +4161306 1234 E-Mail karger@karger.ch www.karger.com
(C) 2010 S. Karger AG, Base

$1420-4096 / 10 / 0331-0001 \$ 26.00 / 0$

Accessible online at:

www.karger.com $/ \mathrm{kbr}$
Dr. Anoop Shankar

Department of Community Medicine

West Virginia University School of Medicine

PO Box 9190, Morgantown, WV 26506-9190 (USA)

Tel. +1 304293 0199, Fax +1 304293 6685, E-Mail ashankar@hsc.wvu.edu 
nationally representative sample of adults in the United States, after adjusting for several important confounders, including alcohol intake.

\section{Methods}

\section{Study Participants}

The NHANES 1999-2002 was a nationally representative sample of the non-institutionalized civilian population of the United States. A detailed summary of the procedures involved in NHANES 1999-2002 has been published and is available online $[10,11]$. In brief, the NHANES survey included a stratified multistage probability sample. Selection was based on counties, blocks, households and individuals within households, and included the oversampling of non-Hispanic Blacks and Mexican-Americans in order to provide stable estimates of these groups. Subjects were required to sign a consent form before their participation, and approval was obtained from the Human Subjects Committee in the US Department of Health and Human Service.

Overall, 11,441 adults $\geq 18$ years of age participated in the interview and examination components of NHANES 1999-2002. Of these participants, serum creatinine and GGT levels are available for 9,836 (85.97\%) participants. Out of these, we further excluded 320 subjects with missing covariable data (e.g., serum cholesterol). This resulted in 9,516 participants (52.4\% women), 622 of whom had CKD.

\section{Main Outcome of Interest: Presence of CKD}

The 4-variable Modification of Diet in Renal Diseases (MDRD) study equation was used to estimate glomerular filtration rate (eGFR) from serum creatinine [12]. CKD was defined as eGFR of $<60 \mathrm{ml} / \mathrm{min} / 1.73 \mathrm{~m}^{2}$, consistent with National Kidney Foundation Kidney Disease Outcomes Quality Initiative (KDOQI) $\geq$ stage 3 CKD [13].

\section{Exposure Measurements}

Age, gender, race/ethnicity, smoking status, alcohol intake (g/day), level of education, history of diabetes and oral hypoglycemic intake or insulin administration, and antihypertensive medication use were assessed using a questionnaire. Individuals who had not smoked $\geq 100$ cigarettes in their lifetimes were considered never smokers; those who had smoked $\geq 100$ cigarettes in their lifetimes were considered former smokers if they answered negatively to the question 'Do you smoke now?' and current smokers if they answered affirmatively. Body mass index (BMI) was calculated as weight in kilograms divided by height in meters squared.

Rigorous procedures with quality control checks were used in blood collection and details about these procedures are provided in the NHANES Laboratory/Medical Technologists Procedures Manual [11]. Serum GGT concentration was assayed with a Hitachi 737 Analyzer (Boehringer-Mannheim Diagnostics, Indianapolis, Ind., USA) at White Sands Research Center, Alamogordo, N. Mex., USA; details of laboratory measurements are available online [10]. Serum total cholesterol was measured enzymatically. Serum glucose was measured using the modified hexokinase method at the University of Missouri Diabetes Diagnostic Laboratory. Diabetes was defined based on the guidelines of the American
Table 1. Baseline characteristics of the study population

\begin{tabular}{lc}
\hline Characteristics & Whole cohort $(\mathrm{n}=9,516)$ \\
\hline Women, \% & $4,986(52.4)$ \\
Age, years & $46.4 \pm 0.3$ \\
$\quad$ Men & $45.0 \pm 0.3$ \\
$\quad$ Women & \\
Race/ethnicity, \% & $4,468(46.9)$ \\
$\quad$ Non-Hispanic Whites & $1,832(19.3)$ \\
$\quad$ Non-Hispanic Blacks & $2,473(25.9)$ \\
$\quad$ Mexican-Americans & $743(7.8)$ \\
$\quad$ Others & \\
Education categories, \% & $3,352(35.2)$ \\
$\quad$ Below high school & $2,280(23.9)$ \\
$\quad$ High school & $3,884(40.8)$ \\
$\quad$ Above high school & \\
Smoking, \% & $5,436(57.1)$ \\
$\quad$ Never smoker & $2,250(23.6)$ \\
$\quad$ Former smoker & $1,830(19.2)$ \\
Current smoker & \\
Alcohol intake, \% & $7,197(75.6)$ \\
$\quad$ Current non-drinker & $2,319(24.4)$ \\
Current drinker & $8.92 \pm 0.3$ \\
Alcohol intake, g & $27.97 \pm 0.1$ \\
Body mass index & $817(9.2)$ \\
Diabetes mellitus, \% & $3,021(31.8)$ \\
Hypertension, \% & $197.21 \pm 0.4$ \\
Total cholesterol, mg/dl & $107.22 \pm 0.4$ \\
Mean GGT, U/l & \\
Mean GFR, ml/min/1.73 m ${ }^{2}$ & \\
$\quad$ &
\end{tabular}

Data presented are number (percentages) or mean values \pm $\mathrm{SE}$, as appropriate for the variable.

Diabetes Association as a serum glucose $\geq 126 \mathrm{mg} / \mathrm{dl}$ after fasting for a minimum of $8 \mathrm{~h}$, a serum glucose $\geq 200 \mathrm{mg} / \mathrm{dl}$ for those who fasted $<8 \mathrm{~h}$ before their NHANES visit, or a self-reported current use of oral hypoglycemic medication or insulin. Seated systolic and diastolic blood pressures were measured using a mercury sphygmomanometer according to the American Heart Association and Seventh Joint National Committee (JNC7) recommendations $[10,14]$. Up to three measurements were averaged for systolic and diastolic pressures. Patients were considered hypertensive if they reported current blood pressure-reducing medication use and/or had systolic blood pressures $\geq 140 \mathrm{~mm} \mathrm{Hg}$ and/or diastolic blood pressures $\geq 90 \mathrm{~mm} \mathrm{Hg}[14]$.

\section{Statistical Analysis}

Serum GGT was analyzed both as a continuous variable and a categorical variable. For the analysis as a continuous variable, serum GGT values were log transformed (base 2) as a result of their skewed distribution. As physiologically normal serum GGT levels vary in men and women, we categorized serum GGT level as quartiles by gender (men: <18, 18-25, 26-38, >38 U/l; women: $<13,13-17,18-25,>25 \mathrm{U} / \mathrm{l})$. 
Table 2. Association between serum GGT levels and CKD

\begin{tabular}{lllll}
\hline $\begin{array}{l}\text { Serum GGT quartiles } \\
\text { in the whole cohort }\end{array}$ & $\begin{array}{l}\text { Number at risk } \\
(\mathrm{n}=9,516)\end{array}$ & $\begin{array}{l}\text { CKD cases } \\
(\mathrm{n}=622)\end{array}$ & $\begin{array}{l}\text { Age-, sex-adjusted OR } \\
(95 \% \mathrm{CI})\end{array}$ & $\begin{array}{l}\text { Multivariable-adjusted OR } \\
(95 \% \text { CI })^{2}\end{array}$ \\
\hline Quartile 1 & 2,364 & 148 & 1 (referent) & 1 (referent) \\
Quartile 2 & 2,555 & 174 & $0.73(0.51,1.02)$ & $0.67(0.49,0.93)$ \\
Quartile 3 & 2,226 & 139 & $0.97(0.72,1.30)$ & $0.94(0.74,1.19)$ \\
Quartile 4 & 2,371 & 161 & $1.01(0.79,1.29)$ & $1.02(0.81,1.28)$ \\
p trend & & 622 & 0.312 & 0.377 \\
log-transformed serum GGT, U/1 & 9,516 & 622 & $0.99(0.84,1.17)$ & $1.01(0.85,1.20)$ \\
\hline
\end{tabular}

${ }^{1}$ GGT quartiles in men, quartile 1-4: $<18,18-25,26-38,>38 \mathrm{U} / 1$, and in women quartile $1-4:<13,13-17,18-25,>25 \mathrm{U} / 1$.

${ }^{2}$ Adjusted for age (years), race-ethnicity (non-Hispanic Whites, non-Hispanic Blacks, Mexican-Americans, others), education categories (below high school, high school, above high school), smoking (never, former, current), alcohol intake (never, former, current), BMI (normal, overweight, obese), diabetes (absent, present), hypertension (absent, present) and serum total cholesterol (mg/dl).

The odds ratio (OR; 95\% confidence interval (CI)) of CKD for each GGT quartile was calculated by taking the lowest quartile as the referent, using multivariable logistic regression models. We used two models: the age- and sex-adjusted model and the multivariable model, additionally adjusting for race/ethnicity (nonHispanic Whites, non-Hispanic Blacks, Mexican-Americans, others), education categories (below high school, high school, above high school), smoking (never smoker, former smoker, current smoker), alcohol intake (g/day), BMI, diabetes mellitus (absent, present), hypertension (absent, present), and total serum cholesterol (mg/dl). Trends in the OR of CKD across increasing serum GGT category were determined modeling GGT categories as an ordinal variable. As GGT levels are related to gender [15], alcohol intake [16] and BMI, [17], subgroup analyses taking these factors into consideration were performed. Also, we performed additional analyses after excluding subjects with self-reported cardiovascular disease and GGT levels indicative of cholestasis ( $>80 \mathrm{U} / \mathrm{l}$ ) to know whether the observed findings remained unchanged. Sample weights that account for the unequal probabilities of selection, oversampling, and non-response were applied for all analyses using SUDAAN (version 8.0; Research Triangle Institute, Research Triangle Park, N.C., USA) and SAS (Version 9.2; SAS Institute, Cary, N.C., USA) software; SEs were estimated using the Taylor series linearization method.

\section{Results}

Among 9,516 adults $\geq 18$ years of age included in the current analyses, 622 (6.53\%) individuals had CKD. Table 1 presents the baseline characteristics of the study population. Mean age was approximately 46 years in men and 45 years in women. Table 2 presents the ORs of CKD by increasing levels of the GGT distribution. There was no association between increasing levels of GGT and $\mathrm{CKD}$ in both the age and sex-adjusted model ( $\mathrm{p}$ trend $=$
Table 3. Association between serum GGT levels and CKD by gender

\begin{tabular}{llll}
\hline $\begin{array}{l}\text { Serum GGT } \\
\text { quartiles }^{1}\end{array}$ & $\begin{array}{l}\text { Number } \\
\text { at risk } \\
(\mathrm{n}=9,516)\end{array}$ & $\begin{array}{l}\text { CKD cases } \\
(\mathrm{n}=622)\end{array}$ & $\begin{array}{l}\text { Multivariable- } \\
\text { adjusted OR } \\
(95 \% \mathrm{CI})^{2}\end{array}$ \\
\hline $\begin{array}{l}\text { Women } \\
\text { Quartile 1 }\end{array}$ & 1,299 & 53 & 1 (referent) \\
$\begin{array}{l}\text { Quartile 2 } \\
\text { Quartile 3 }\end{array}$ & 1,286 & 90 & $0.70(0.44,1.11)$ \\
$\begin{array}{l}\text { Quartile 4 } \\
\text { p trend }\end{array}$ & 1,152 & 85 & $0.97(0.71,1.32)$ \\
log-transformed & 1,249 & 101 & $0.96(0.78,1.18)$ \\
$\quad$ & & & 0.498 \\
$\quad$ serum GGT, U/1 & 4,986 & 329 & $0.95(0.78,1.16)$ \\
\hline $\begin{array}{l}\text { Men } \\
\text { Quartile 1 }\end{array}$ & 1,065 & 95 & $1($ referent $)$ \\
$\begin{array}{l}\text { Quartile 2 } \\
\text { Quartile 3 }\end{array}$ & 1,269 & 84 & $0.65(0.41,1.03)$ \\
$\begin{array}{l}\text { Quartile 4 } \\
\text { p trend } \\
\text { log-transformed }\end{array}$ & 1,074 & 54 & $0.89(0.64,1.24)$ \\
$\quad$ serum GGT, U/1 & 4,530 & 293 & $1.04(0.82,1.32)$ \\
\hline
\end{tabular}

${ }^{1}$ GGT quartiles in men, quartile $1-4:<18,18-25,26-38,>38$ $\mathrm{U} / \mathrm{l}$, and in women quartile $1-4:<13,13-17,18-25,>25 \mathrm{U} / \mathrm{l}$.

${ }^{2}$ Adjusted for age (years), race-ethnicity (non-Hispanic Whites, non-Hispanic Blacks, Mexican-Americans, others), education categories (below high school, high school, above high school), smoking (never, former, current), alcohol intake (never, former, current), BMI (normal, overweight, obese), diabetes (absent, present), hypertension (absent, present) and serum total cholesterol (mg/dl). 
Table 4. Association between increasing serum GGT levels and CKD by alcohol intake status

\begin{tabular}{|c|c|c|c|c|}
\hline \multirow{2}{*}{$\begin{array}{l}\text { Serum GGT } \\
\text { quartiles cohort }^{1}\end{array}$} & \multicolumn{2}{|c|}{ Current non-drinker $(\mathrm{n}=7,197)$} & \multicolumn{2}{|c|}{ Current drinker $(\mathrm{n}=2,319)$} \\
\hline & $\begin{array}{l}\text { number at risk } \\
\text { (CKD cases) }\end{array}$ & $\begin{array}{l}\text { multivariable OR } \\
(95 \% \mathrm{CI})^{2}\end{array}$ & $\begin{array}{l}\text { number at risk } \\
\text { (CKD cases) }\end{array}$ & $\begin{array}{l}\text { multivariable OR } \\
(95 \% \mathrm{CI})^{2}\end{array}$ \\
\hline Quartile 1 & $1,921(122)$ & 1 (referent) & $443(26)$ & 1 (referent) \\
\hline Quartile 2 & $1,936(146)$ & $0.77(0.56,1.05)$ & $619(28)$ & $0.63(0.21,1.89)$ \\
\hline Quartile 3 & $1,653(123)$ & $0.85(0.62,1.15)$ & $573(16)$ & $0.97(0.46,2.04)$ \\
\hline Quartile 4 & $1,687(133)$ & $0.96(0.62,1.49)$ & $684(28)$ & $1.01(0.62,1.64)$ \\
\hline $\mathrm{p}$ trend & & 0.378 & 0.6024 & 0.627 \\
\hline
\end{tabular}

${ }^{1}$ GGT quartiles in men, quartile $1-4:<18,18-25,26-38,>38 \mathrm{U} / 1$, and in women quartile $1-4:<13,13-17$, $18-25,>25 \mathrm{U} / 1$.

${ }^{2}$ Adjusted for age (years), sex (men, women), race-ethnicity (non-Hispanic Whites, non-Hispanic Blacks, Mexican-Americans, others), education categories (below high school, high school, above high school), smoking (never, former, current), BMI (normal, overweight, obese), diabetes (absent, present), hypertension (absent, present) and serum total cholesterol (mg/dl).

Table 5. Association between increasing serum GGT levels and CKD by BMI

\begin{tabular}{|c|c|c|c|c|}
\hline \multirow{2}{*}{$\begin{array}{l}\text { Serum GGT } \\
\text { quartiles cohort }^{1}\end{array}$} & \multicolumn{2}{|c|}{ Normal weight $(\mathrm{n}=3,326)$} & \multicolumn{2}{|c|}{ Overweight/obese $(n=6,190)$} \\
\hline & $\begin{array}{l}\text { number at risk } \\
\text { (CKD cases) }\end{array}$ & $\begin{array}{l}\text { multivariable OR } \\
(95 \% \mathrm{CI})^{2}\end{array}$ & $\begin{array}{l}\text { number at risk } \\
\text { (CKD cases) }\end{array}$ & $\begin{array}{l}\text { multivariable OR } \\
(95 \% \mathrm{CI})^{2}\end{array}$ \\
\hline \multicolumn{5}{|l|}{ Whole cohort } \\
\hline Quartile 1 & $1,264(64)$ & 1 (referent) & $1,100(84)$ & 1 (referent) \\
\hline Quartile 2 & $969(43)$ & $0.56(0.38,0.84)$ & $1,586(131)$ & $1.06(0.52,2.19)$ \\
\hline Quartile 3 & $596(51)$ & $0.66(0.45,0.96)$ & $1,630(88)$ & $0.98(0.50,1.92)$ \\
\hline Quartile 4 & $497(32)$ & $1.04(0.57,1.89)$ & $1,874(129)$ & $0.87(0.40,1.92)$ \\
\hline $\mathrm{p}$ trend & & 0.418 & & 0.958 \\
\hline
\end{tabular}

${ }^{1}$ GGT quartiles in men, quartile $1-4:<18,18-25,26-38,>38 \mathrm{U} / 1$, and in women quartile $1-4:<13,13-17$, $18-25,>25 \mathrm{U} / 1$.

${ }^{2}$ Adjusted for age (years), sex (men, women), race-ethnicity (non-Hispanic Whites, non-Hispanic Blacks, Mexican-Americans, others), education categories (below high school, high school, above high school), smoking (never, former, current), alcohol intake (never, former, current), diabetes (absent, present), hypertension (absent, present) and serum total cholesterol (mg/dl).

$0.312)$ and the multivariable adjusted models ( $\mathrm{p}$ trend $=$ 0.377 ), and the magnitude of OR hovered around 1, consistent with a null effect. This relationship persisted even when serum GGT was analyzed as a continuous variable.

In table 3, we present the gender-specific analyses for the association between increasing GGT levels and CKD. Here also, consistent with the findings for the whole cohort, there was no clear association between increasing GGT levels and CKD either among men $(\mathrm{p}$ trend $=0.668)$ or among women ( $\mathrm{p}$ trend $=0.498$ ).
Tables 4 and 5 examined the association between increasing serum GGT levels and CKD within subgroups of alcohol intake (current non-drinker, current drinker) and BMI (normal weight, overweight/obese). Consistent with findings for the whole cohort, there was no clear association between increasing GGT levels and CKD, either among current non-drinkers ( $p$ trend $=0.378$ ) or current drinkers ( $\mathrm{p}$ trend $=0.627$ ), or among normal weight subjects ( $p$ trend $=0.418$ ) or overweight/obese subjects ( $\mathrm{p}$ trend $=0.958)$. 
We performed several supplementary analyses to confirm the robustness of our findings. First, we repeated the main analysis after excluding subjects with history of cardiovascular disease; the results were essentially the same. Compared to quartile 1 of GGT, the multivariable OR (95\% CI) of CKD was $0.75(0.44-1.28)$ for quartile 2, 1.03 (0.63-1.68) for quartile 3, $0.96(0.58-1.59)$ for quartile 4 ; $\mathrm{p}$ trend $=0.541$. Second, we repeated the main analysis after excluding subjects with very high serum GGT levels ( $>80 \mathrm{U} / \mathrm{l})$, indicative of cholestasis; here also the results were similar to our main findings. Compared to quartile 1 of GGT, the multivariable OR $(95 \% \mathrm{CI})$ of CKD was 0.87 $(0.52-1.45)$ for quartile $2,0.96(0.70-1.32)$ for quartile 3 , $0.91(0.76-1.09)$ for quartile 4 ; $\mathrm{p}$ trend $=0.392$.

\section{Discussion}

In our analysis of a nationally representative sample of US adults, we found no association between increasing GGT levels and CKD. The magnitude of ORs for the association between increasing quartiles of GGT and CKD was consistent with an overall null association when adjusted initially for age, sex, race/ethnicity, and then further for education, smoking, alcohol intake, BMI, diabetes, hypertension, and total cholesterol. Subsequent subgroup analyses by gender, alcohol intake, and BMI categories yielded similar results. In subsidiary analyses that excluded subjects with cardiovascular disease and cholestasis from the study population, the results yet again showed no discernable association over the range of serum GGT quartiles and CKD.

Previous studies have shown that higher GGT levels in serum predicted the risk of cardiovascular disease $[6,18]$. Studies have also shown that serum GGT levels are related to diabetes mellitus [7] and hypertension [8,9] which are strong, independent risk factors for CKD. However, few studies have examined the putative association between serum GGT and CKD.

Ryu et al. [19] in a recent prospective study from Korea reported a positive association between serum levels of GGT and CKD. They found that the association between GGT and CKD was independent of factors such as diabetes and hypertension. However, this study included only men and examined an occupational cohort of workers from a semiconductor manufacturing plant. It is not clear if these results are also applicable to women. Second, the fact that they studied a cohort of healthy workers and not a community-based sample could have biased their findings (e.g. healthy worker effect). Third, as GGT levels are influenced by alcohol and diet, findings from Asian populations may not be directly applicable to Western populations with different alcohol intake and dietary patterns.

In this context, the current study is the first report on the association between serum GGT and CKD in a large sample of US adults. It is worth noting that in contrast to the Korean study [19], we examined both men and women, and studied a wider range of alcohol intake patterns, representative of typical Western populations. Our results, however, indicate no association between increasing serum GGT levels and CKD. The large sample size available for the current analyses, our ability to adjust for all potential confounders, and the consistency of these results of an overall null association suggest that our findings are less likely due to chance. Furthermore, the observed null association was consistently present in supplementary analyses where we excluded subjects with cardiovascular disease and high GGT levels indicative of cholestasis.

The key strengths of our study include the following. As we examined a representative sample of US adults, these results are generalizable to US adults. Furthermore, all data were collected following rigorous methodology, including a study protocol with standardized quality control checks [11]. The main limitation of our study is the cross-sectional nature of NHANES, which precludes conclusions regarding the temporal nature of the association between serum GGT and CKD. Also, another study limitation that could have biased our results is the lack of information on waist circumference or visceral fat accumulation as a measure of obesity.

In conclusion, we found no association between increasing serum GGT levels and CKD in a sample of US adults. These findings suggest that the previously reported association between serum GGT and CKD may be related to confounding factors such as gender, alcohol intake, BMI, diabetes, and hypertension. Our study does not support measuring serum GGT for the purpose of CKD prediction in Western populations.

\section{Acknowledgements}

This study was funded by a Research Funding Development Grant (RFDG) and start-up funds to A.S. from the West Virginia University School of Medicine. 


\section{References}

1 Whitfield JB: Gamma-glutamyl transferase. Crit Rev Clin Lab Sci 2001;38:263-355.

$\checkmark 2$ Heisterkamp N, Groffen J, Warburton D, Sneddon TP: The human $\gamma$-glutamyltransferase gene family. Hum Genet 2008;123: 321-332.

$\checkmark 3$ Whitfield JB: Serum $\gamma$-glutamyltransferase and risk of disease. Clin Chem 2007;53:1-2.

$\checkmark 4$ Song SH, Kwak IS, Kim YJ, et al: Can $\gamma$-glutamyltransferase be an additional marker of arterial stiffness? Circ J 2007;71:1715-1720.

$\checkmark 5$ Shankar A, Li J, Klein BE, Javier NF, Klein R: Serum $\gamma$-glutamyltransferase level and peripheral arterial disease. Atherosclerosis 2008;199:102-109.

-6 Ruttmann E, Brant LJ, Concin H, Diem G, Rapp K, Ulmer H: Gamma-glutamyltransferase as a risk factor for cardiovascular disease mortality: an epidemiological investigation in a cohort of 163,944 Austrian adults. Circulation 2005;112:2130-2137.

$\checkmark 7$ Perry IJ, Wannamethee SG, Shaper AG: Prospective study of serum $\gamma$-glutamyltransferase and risk of NIDDM. Diabetes Care 1998; 21:732-737.
Lee DH, Jacobs DR Jr, Gross M, et al: Gamma-glutamyltransferase is a predictor of incident diabetes and hypertension: the Coronary Artery Risk Development in Young Adults (CARDIA) study. Clin Chem 2003; 49:1358-1366.

9 Shankar A, Li J: Association between serum $\gamma$-glutamyltransferase level and prehypertension among US adults. Circ J 2007;71: 1567-1572.

10 National Center for Health Statistics: NHANES 1999-2002 Survey Questionnaires ECaLC. Available at: www.cdc.gov/ nchs/about/major/nhanes/questexam01_ 02.htm (accessed: Nov 28 2008), 2008.

11 National Center for Health Statistics: NHANES 1999-2002 addendum to NHANES III analytic guidelines. Available at: www.cdc.gov/nchs/data/nhanes/guidelines1.pdf (accessed: Nov 29, 2008), 2002.

12 Levey AS, Coresh J, Greene T, et al: Using standardized serum creatinine values in the Modification of Diet in Renal Disease study equation for estimating glomerular filtration rate. Ann Intern Med 2006;145:247254.

$13 \mathrm{~K} / \mathrm{DOQI}$ clinical practice guidelines for chronic kidney disease: evaluation, classification, and stratification. Am J Kidney Dis 2002;39(suppl 1):S1-S266.
14 Chobanian AV, Bakris GL, Black HR, et al: Seventh report of the Joint National Committee on Prevention, Detection, Evaluation, and Treatment of High Blood Pressure. Hypertension 2003;42:1206-1252.

15 Carulli L, Lonardo A, Lombardinil S, Marchesini G, Loria P: Gender, fatty liver and GGT. Hepatology 2006;44:278-279.

16 Lee DH, Ha MH, Kim JR, Gross M, Jacobs DR Jr: Gamma-glutamyltransferase, alcohol, and blood pressure. A four-year follow-up study. Ann Epidemiol 2002;12:90-96.

17 Puukka K, Hietala J, Koivisto H, Anttila P, Bloigu R, Niemela O: Additive effects of moderate drinking and obesity on serum $\gamma$ glutamyltransferase activity. Am J Clin Nutr 2006;83:1351-1354.

18 Lee DS, Evans JC, Robins SJ, et al: Gammaglutamyl transferase and metabolic syndrome, cardiovascular disease, and mortality risk: the Framingham Heart Study. Arterioscler Thromb Vasc Biol 2007;27:127133.

19 Ryu S, Chang Y, Kim DI, Kim WS, Suh BS: Gamma-glutamyltransferase as a predictor of chronic kidney disease in nonhypertensive and nondiabetic Korean men. Clin Chem 2007;53:71-77. 